\title{
Hyperplastic Nodular Hepatic Lesions Following End-to- side Portacaval Shunting in Childhood
}

\author{
Nobuyoshi Fukushima ${ }^{1}$, Ryoko Kuromatsu ${ }^{1}$, Daiji Uchiyama ${ }^{2}$, Satoshi Itano ${ }^{1,3}$, Akio Takata ${ }^{1}$, \\ Eiji Ando ${ }^{1}$, Shuji Sumie ${ }^{1}$, Takuji Torimura ${ }^{1}$, Masafumi Uchida ${ }^{2}$, Osamu Nakashima ${ }^{4}$, \\ Masamichi Kojiro ${ }^{4}$ and Michio Sata ${ }^{1}$
}

\begin{abstract}
We describe a 48-year-old man with nodular intrahepatic lesions accompanied by communication between the inferior vena cava and portal systems as well as absence of intrahepatic portal veins. After infection with malaria in childhood, end-to-side portacaval shunting had been performed to treat upper gastrointestinal bleeding at the age of 15 years. A biopsy specimen obtained under ultrasonographic guidance showed hyperplastic nodules suggestive of focal nodular hyperplasia. The estradiol concentration in the blood was elevated $(55 \mathrm{pg} / \mathrm{ml})$. This case suggests that portacaval shunting may be associated with hyperplastic liver nodules through hyperestrogenemia and abnormal hepatic hemodynamics.
\end{abstract}

Key words: portacaval anastomosis, focal nodular hyperplasia, hyperestrogenemia

(DOI: 10.2169/internalmedicine.46.6419)

\section{Introduction}

End-to-side portacaval anastomosis, termed an Eck fistula $(1,2)$, is an operation performed to treat life-threatening portal hypertension by creating a major shunt between the inferior vena cava (IVC) and the portal trunk. The structural relationship between portal and systemic veins after portacaval shunting is similar to that seen in congenital absence of the portal vein (CAPV), which often is associated with hyperplastic liver lesions. Development of such lesions following a portacaval shunt procedure has been reported only in patients with type I glycogen storage disease (GSD-I). The present case appears to be the first occurrence of benign hyperplastic liver lesions to follow portosystemic shunting in a patient with no other condition predisposing to hepatic neoplasia.

\section{Case Report}

A 48-year-old Japanese man was referred to our institu- tion because of nodular intrahepatic lesions recently detected ultrasonographically at another hospital. He was infected with malaria (from his uncle) in childhood and received medication. No further details regarding his medication were available. Splenectomy was performed when the patient was 5 years old. At 15 years old hematemesis occurred, most likely a result of portal hypertension. A shunt operation was performed, and a blood transfusion was given. Earlier abdominal ultrasonography, performed 13 months before referral to our hospital, had shown no nodular lesion in the liver.

On admission to our hospital, his performance status was excellent. No symptoms of malaria were evident. The liver was not palpable. A healed midline abdominal scar was noted. The patient's history disclosed only minimal alcohol intake. Serologic and molecular tests for hepatitis B and C viruses (HBV and $\mathrm{HCV}$ ) indicated past infection by both viruses [HB surface antigen negative, HB core antibody positive, HCV antibody positive, HCV-RNA negative]. Serum chemistry data showed mild elevations of aspartate aminotransferase (AST, $42 \mathrm{U} / \mathrm{L}$; normal, 13 to 33), gammaglutamyl transpeptidase ( $\gamma$-GTP, $81 \mathrm{U} / \mathrm{L}$; normal, 10 to 47 ),

\footnotetext{
${ }^{1}$ Division of Gastroenterology, Department of Medicine, Kurume University School of Medicine, Kurume, ${ }^{2}$ Departments of Radiology, Kurume University School of Medicine, Kurume, ${ }^{3}$ Kurume Chuo Hospital, Kurume and ${ }^{4}$ Departments of Pathology, Kurume University School of Medicine, Kurume

Received for publication December 8, 2006; Accepted for publication May 8, 2007

Correspondence to Dr. Nobuyoshi Fukushima, kingsno1@ @med.kurume-u.ac.jp
} 
lactate dehydrogenase (LDH, $331 \mathrm{U} / \mathrm{L}$; normal, 119 to 229) and hyaluronic acid $(59 \mathrm{ng} / \mathrm{ml}$; normal, $\leqq 50)$. Albumin was slightly low (3.49 $\mathrm{g} / \mathrm{dl}$; normal, 4.0 to 5.0$)$. Indocyanine green retention (ICG $\mathrm{R}_{15}$ ) was $55.3 \%$ (normal, $\leqq 10$ ), while ammonia $\left(\mathrm{NH}_{3}\right)$ was $135 \mu \mathrm{g} / \mathrm{dl}$ (normal, 12 to 66 ).

Abdominal ultrasonography showed evidence of chronic liver damage and steatosis, as well as iso- to hypoechoic nodule in segment VI $(20 \times 21 \mathrm{~mm}$ in diameter) (Fig. 1). Three smaller nodules were present $(7 \times 8 \mathrm{~mm}$ in segment VI, $6 \times 8 \mathrm{~mm}$ in segment VI, and $10 \times 10 \mathrm{~mm}$ in segment VII). Color Doppler ultrasonography revealed an artery branching from an artery at the margin of the largest nodule, then approaching the center of the nodule. No "spokewheel" pattern was seen (Fig. 2). Dynamic computed tomography (CT) detected only the largest nodule, which was isodense prior to contrast agent administration and in the delayed phase, showing mild enhancement at the early phase (Fig. 3A, B). Portal flow within the liver or in the portal tract approaching the hepatic hilum was not detected (Fig. 3C). The IVC communicated with the superior mesenteric vein (SMV) and the narrow splenic vein (SV). A normal IVC was not seen inferior to its connection with the SMV and SV; instead, two prominent veins likely to be the left IVC and the testicular vein drained into the left renal vein from below. The complexity of portal and systemic venous anatomy were shown clearly by three-dimensional
(3D) CT (Fig. 4). Magnetic resonance imaging (MRI) showed the largest hepatic nodule to be hyperintense on both T1-weighted and T2-weighted images. Superparamagnetic iron oxide (SPIO)-enhanced MRI using Resovist as a

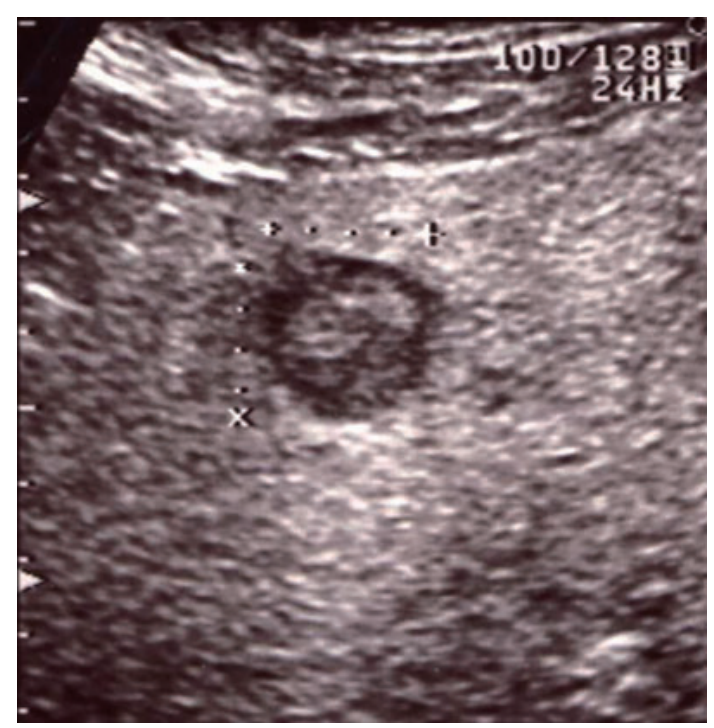

Figure 1. Ultrasonogram showed an iso- to hypoechoic nodule in segment VI. This largest nodule measured $20 \times 21 \mathrm{~mm}$. The echo pattern in surrounding liver parenchyma demonstrated fatty liver and associated chronic damage.

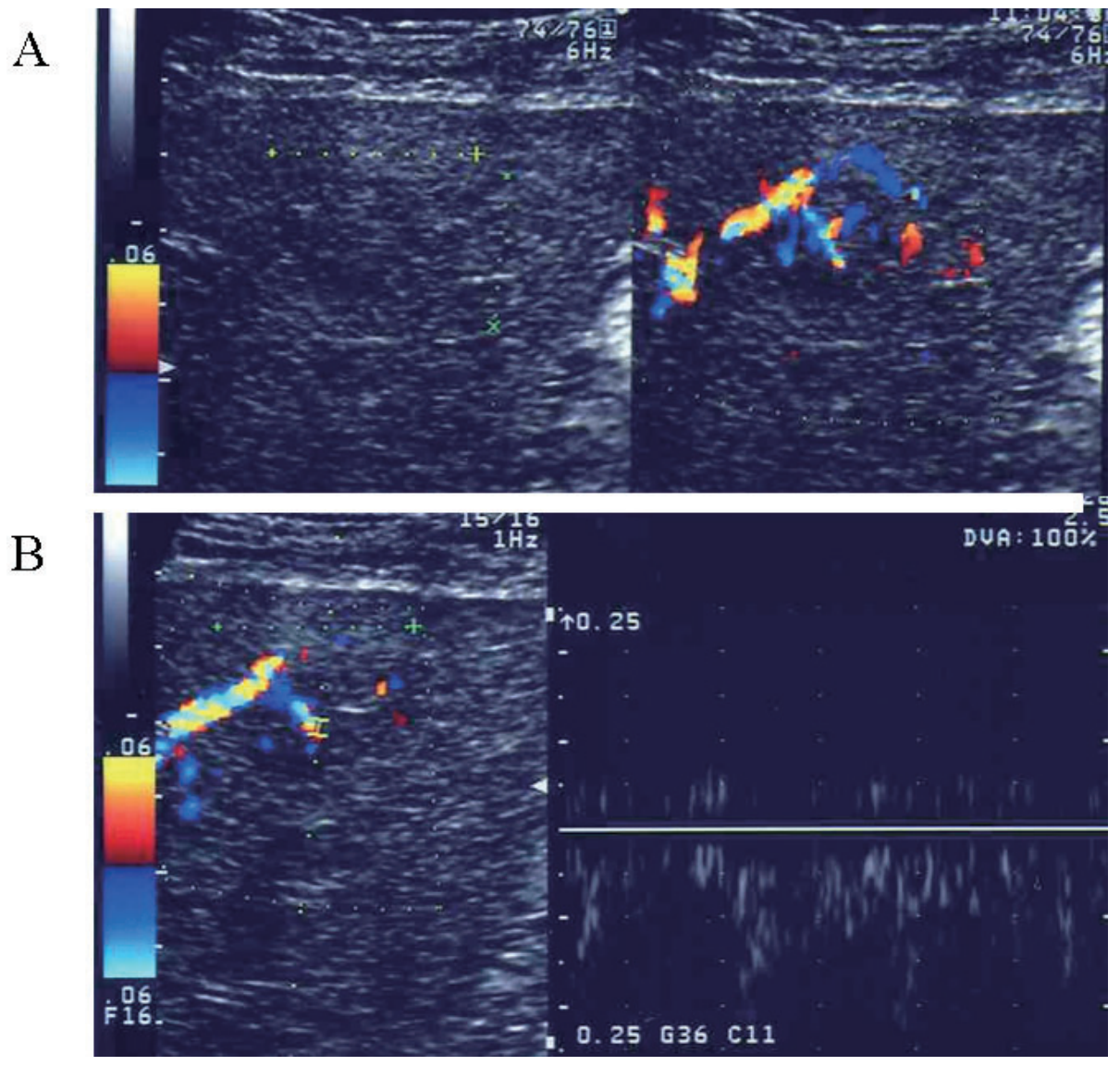

Figure 2. Color Doppler examination. A: Color Doppler image showed an artery running along the margin of nodule, with a branch turning toward the center of the nodule. B: Fast Fourier transformation indistinctly demonstrated an arterial pulsatile wave in this branched artery. 
A

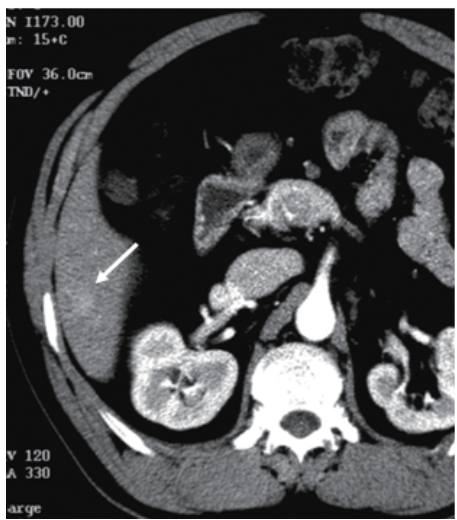

$\mathrm{B}$

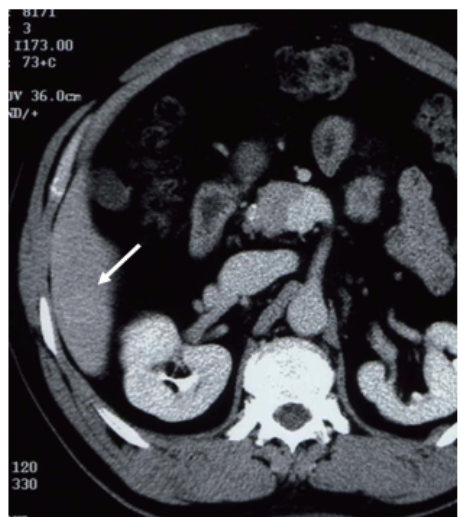

C

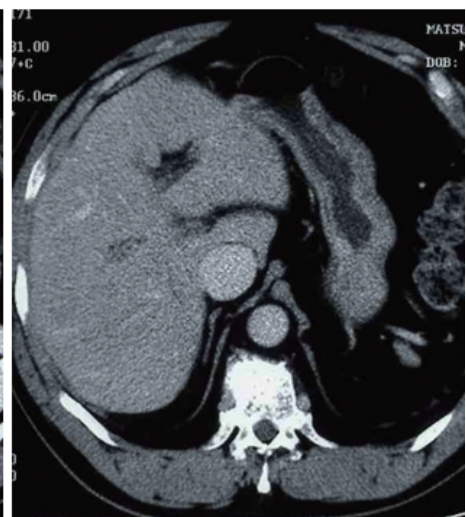

Figure 3. Abdominal computed tomography. A: The largest nodule showed contrast enhancement in the early phase (arrow). B: The largest nodule appeared isodense in the delayed phase (arrow). C: No portal flow is shown in the liver (delayed phase).

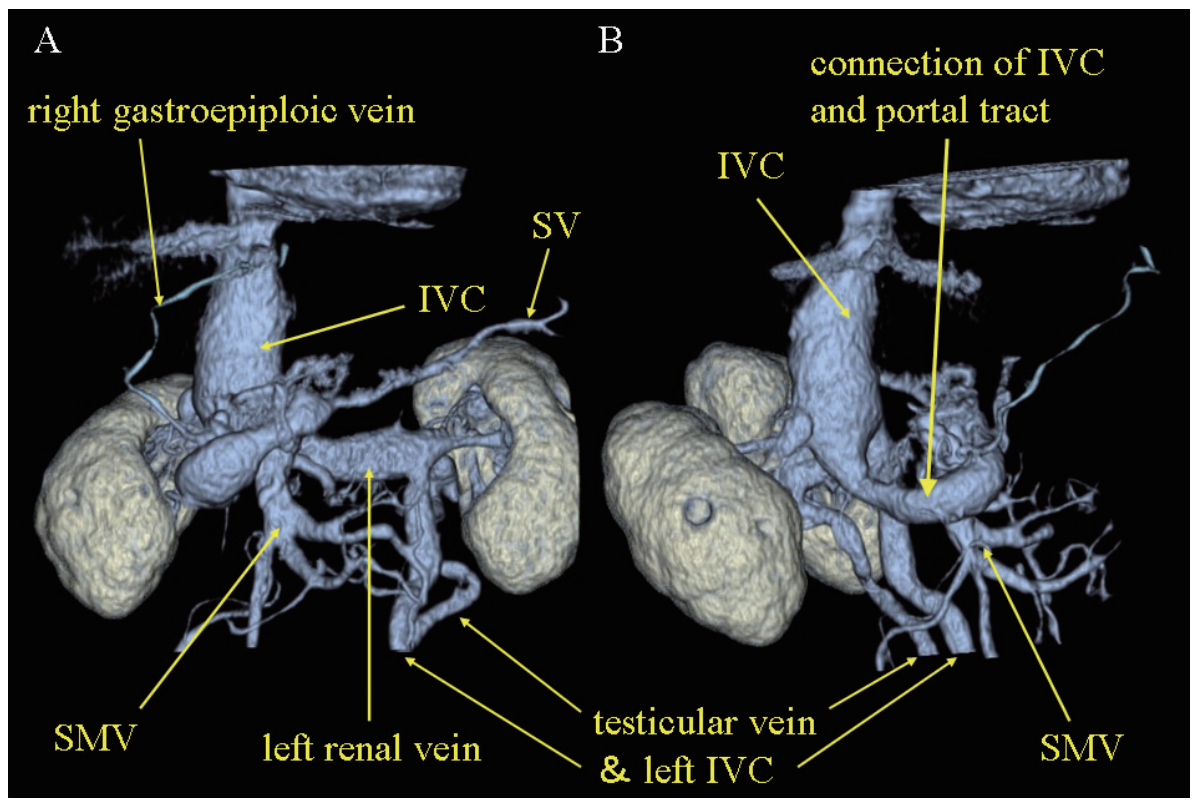

Figure 4. Three-dimensional computed tomography of the venous system. The inferior vena cava (IVC) communicated with the superior mesenteric vein (SMV) and the splenic vein (SV). The IVC disappeared below the level of its connection with the SMV and SV. Two veins, apparently a left IVC and the testicular vein, flowed into the left renal vein. A: Anterior view. B: Right lateral view. IVC: the inferior vena cava, SMV: the superior mesenteric vein, SV: the splenic vein.

contrast agent showed the largest nodule to be hyperintense relative to surrounding liver, indicating less accumulation of Resovist in the nodule (Fig. 5).

A biopsy specimen from the largest nodule consisted of tissue from the nodular lesion and also from surrounding liver (Fig. 6A). Histopathologically, the nodule showed mildly increased cellularity with distinct trabecular architecture, and occasionally a pseudoglandular pattern. Surrounding liver tissue showed mild fatty change. A specimen from a smaller nodule was similar to the trabecular areas of the larger nodule. Both nodules contained areas of fibrous scarring, with a few anomalous arterial vessels and biliary pseudoductules (Fig. 6B). Immunohistochemically, nodule tissue was strongly reactive to anti-CD34 along sinusoids, while surrounding liver tissue was not (Fig. 6C). Cells reactive to anti-CD68 along a nodule sinusoid were fewer than in surrounding liver (Fig. 6D). The nodules were diagnosed as showing the morphology of focal nodular hyperplasia (FNH). We decided to maintain outpatient clinical follow-up as opposed to treatment for tumors such as resection.

\section{Discussion}

We could not obtain any detailed early-life medical records concerning the patient's infection with malaria, splenectomy or shunt operation. Based upon radiologic findings 
and any past history that the patient and his mother could remember, we concluded that somehow he was infected with malaria from his uncle, then underwent splenectomy as a treatment related to malaria. End-to-side portacaval shunting was carried out as treatment for portal hypertension conse-

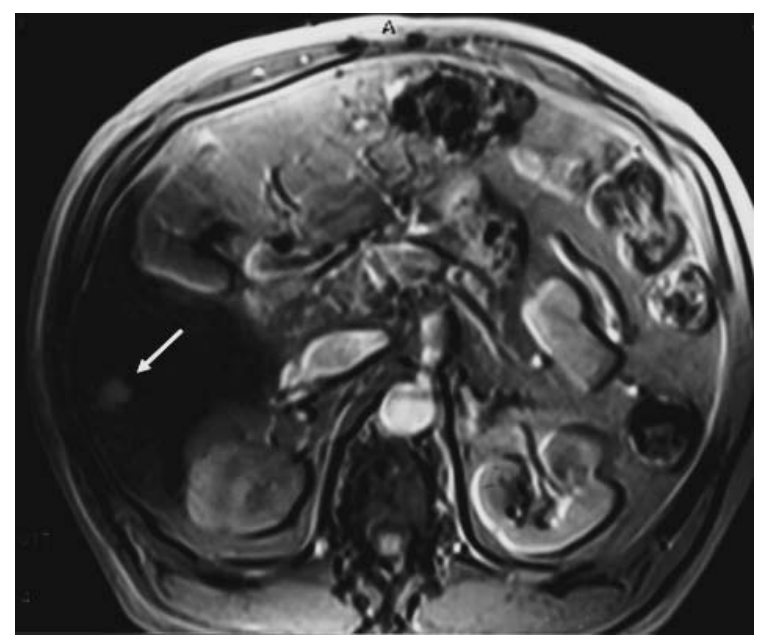

Figure 5. Magnetic resonance imaging using superparamagnetic iron oxide (Resovist). This enhanced imaging showed the largest nodule to be hyperintense relative to surrounding liver (arrow). quent to malaria or to portal thrombosis after splenectomy. Antibodies characteristic of malarial infection were negative, and no organisms could be found in blood smears when the patient was admitted, most likely because the infection was remote.

Paucity of intrahepatic portal blood flow resulted from the shunt operation at age 15. Images from 3D-CT suggested the absence of the IVC below its junction with the left renal vein was congenital. Lack of intrahepatic portal blood flow and radiologic configurations of the portal and systemic veins in the present patient resembled those of congenital absence of the portal vein (CAPV) type Ib (liver not perfuse with portal blood, while the SMV and SV form a confluence) $(3,4)$. Although our patient was not examined by angiography, 3D-CT clearly depicted the complexities of the abnormal abdominal vascular anatomy.

Biopsy specimens from nodular hepatic lesions in the present case showed marked hepatocytic hyperplasia with an area of fibrosis scarring containing anomalous vessels and showing marked sinusoidal capillarization as seen in FNH. Hirohashi et al demonstrated that FNH included capillarized sinusoids (5), while Tanaka et al showed that $15 \%$ of FNH cases showed fewer Kupffer cells in nodules than in surrounding liver tissue (6). Imaging findings in the largest nodule closely depicted the pathologic findings. Hypervascu-
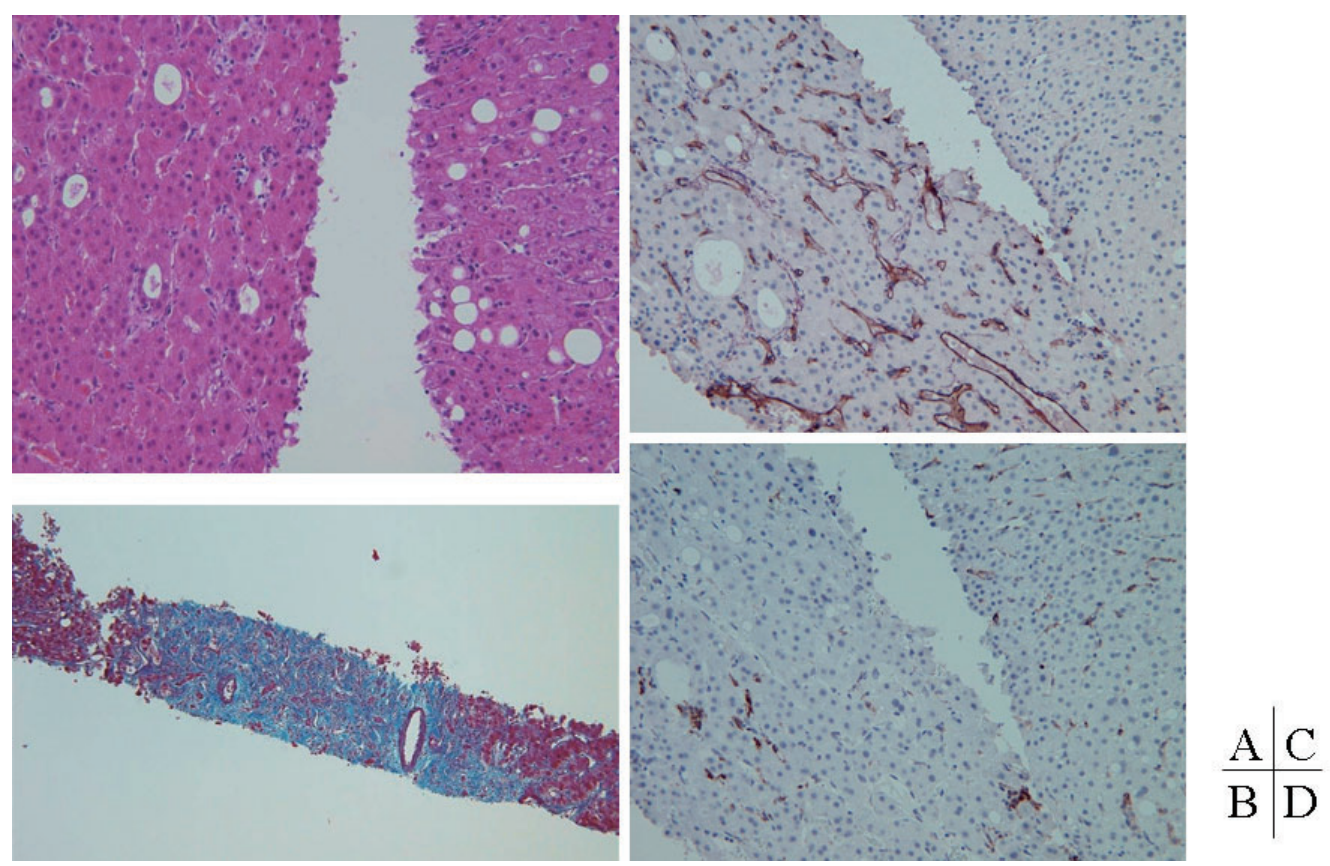

Figure 6. Pathologic findings in biopsy specimens. A: A specimen from the largest nodule $(20 \times 21$ $\mathrm{mm}$, in segment IV) consisted of tissue from the nodule (left) and surrounding liver (right). Tissue from the nodule showed mildly increased cellularity, a distinct trabecular pattern, and occasional pseudoglands. The surrounding liver showed mild fatty change (hematoxylin and eosin stain, $\times 200)$. B: A specimen from a smaller nodule $(7 \times 8 \mathrm{~mm}$, in segment IV) contained a fibrous scar with anomalous arterial blood vessels (Azan stain, $\times 100$ ). C: Immunostaining with anti-CD34 showed a strongly positive reaction in sinusoidal endothelial cells (capillarization) in tissue from the largest nodule (left), exceeding the reactivity in the surrounding tissue (right)(×200). D: Immunostaining with anti-CD68 demonstrated fewer positive cells in the largest nodule (left) than in the surrounding liver (right) $(\times 200)$. 
larity seen by dynamic CT corresponded to anomalous arterial vessels in a fibrous scar, as well as marked sinusoidal capillarization. Findings by SPIO-enhanced MRI are explained by a decrement of CD-68-positive cells, possibly Kupffer cells, along the sinusoids. Accordingly, the nodules were highly suggestive of multiple FNH lesions.

Various hyperplastic liver lesions have been associated with portal venous abnormalities, and frequently occurring in association with CAPV (7), a rare malformation seen mostly in children. Reviewing the literature concerning CAPV, De Gaetano et al found that 15 of 31 cases had hepatic tumors (7): hepatoblastoma in $1, \mathrm{FNH}$ in 9, adenoma in 1, nodular regenerative hyperplasia (NRH) in 1, hemangioma in 1, and hepatocellular carcinoma (HCC) in 2. Additionally, 2 cases of CAPV with $\mathrm{FNH}(8,9), 1$ case with HCC (10), 3 cases with NRH $(11,12)$, and 3 cases with hyperplastic nodular hepatic lesions (13-15) recently were reported. Hemodynamic imbalance with insufficient blood supply and nonuniform arterial perfusion to the liver would appear likely to induce hyperplasia in livers of patients with CAPV $(16,17)$. In addition, the portal vein carries substances in splanchnic venous blood such as insulin, glucagon, epidermal growth factor and other hepatotrophic peptides. As these substances regulate hepatocytic function and development, diversion of portal flow may induce abnormalities by causing dysregulation $(18,19)$.

Alteration of portal and systemic venous structure thus would appear to be a likely cause of hyperplasia of hepatocytes in the present case, as in the occurrence of hyperplasic liver lesions in patients with CAPV. Grün et al reported that portacaval anastomosis in rats resulted in hyperestrogenemia and hypoandrogenemia, while the occurrence of FNH also has been observed (20). In the present case testosterone was in the normal range $(5.30 \mathrm{ng} / \mathrm{ml}$; normal, 2.07 to 7.61$)$, while estradiol was elevated (55 pg/ml; normal, 15 to 35 ). Elevated estrogen in the systemic circulation after portacaval shunting may induce formation of hyperplastic nodular lesions in the liver. The observed association of FNH with oral contraceptive use (21), as well as estrogen receptor expression in mammalian hepatocytes (22) would support this speculation.

While the occurrence of hyperplastic liver lesions includ- ing FNH has been observed in animal model after experimental portacaval shunting $(20,23)$, few reports have described benign hyperplastic lesions in livers of a patient with portacaval anastomosis. FNH has been reported in nine patients with type I glycogen storage disease (GSD-I), while five of these nine patients had been treated with a portacaval shunt $(24,25)$. Takamura et al (24) suggested that portacaval shunts favored the development of FNH in livers of the patients with GSD-I, an inherited disorder frequently accompanied by hepatic tumors (mainly adenomas). Additionally, several studies suggested that portasystemic shunt was associated with a higher risk of developing HCC in the patients with cirrhosis $(26,27)$. From such previous literature portacaval anastomosis is considered to promote induction of tumor in the liver essentially inclined to develop liver tumor, for example cirrhotic liver or liver of patient with GSD-I. Webster et al (28) reported a hepatic adenoma that became greater after mesocaval shunt for portal venous obstruction, suggesting that portasystemic shunt promotes the development of hyperplastic lesion that already existed in the liver. The liver of present patient was not cirrhotic, Budd-Chiari syndrome and GSD-I were also excluded, and earlier abdominal ultrasonography had shown no nodular lesion in the liver. Thus, our case suggested that portasystemic shunt may produce a tumor in a liver with originally no tumor, and in the liver of a patient with no inclination to develop hepatic neoplasm. Previous malaria infection might have been assosiated with the occurrence of hyperplastic lesion in the present case. However, there is no report of hepatic tumor of a patient with malaria, only fibrosing necrotic nodule has been reported (29). One study suggested a negative correlation of malaria with HCC (30).

Our report appears to be the first to describe benign hyperplastic lesions in the human liver following portosystemic anastomosis, except for patients with GSD-I. This case suggests that iatrogenic changes in the portal and systemic venous systems can result in hyperestrogenemia and disappearance of intrahepatic portal flow to cause hyperplastic nodule formation in a previously normal liver. This case supports the theory that abnormality of intrahepatic blood flow initiates hyperplasia of hepatocytes which is often observed in the liver of patients with CAPV.

\section{References}

1. Hallett EB, Holton GW, Paterson JC, Schilling JA. Liver blood and following Eck fistula; liver blood flow before and after splenectomy. Surg Gynecol Obstet 95: 401-406, 1952.

2. Blakemore AH, Lord JW. A non-suture method of blood vessel anastmosis: review of experimental study. Reports of clinical cases. Ann Surg 121: 435-453, 1945.

3. Morgan G, Superina R. Congenital absence of the portal vein: two cases and a proposed classification system for portasystemic vascular anomalies. J Pediatr Surg 29: 1239-1241, 1994.

4. Howard ER, Davenport M. Congenital extrahepatic portocaval shunts-the Abernethy malformation. J Pediatr Surg 32: 494-497, 1997.

5. Hirohashi K, Yamamoto T, Tanaka S, et al. Immunohistochemical demonstration of sinusoidal capillarization in human benign liver tumors: distinction from neoangiogenesis. Hepatol Res 28: 87-93, 2004.

6. Tanaka M, Nakashima O, Wada Y, Kage M, Kojiro M. Pathomorphological study of Kupffer cells in hepatocellular carcinoma and hyperplastic nodular lesions in the liver. Hepatology 24: 807-812, 1996.

7. De Gaetano AM, Gui B, Macis G, Manfredi R, Di Stasi C. Congenital absence of the portal vein associated with focal nodular hyperplasia in the liver in an adult woman: imaging and review of the literature. Abdom Imaging 29: 455-459, 2004.

8. Schmidt S, Saint-Paul MC, Anty R, Bruneton JN, Gugenheim J, Chevallier P. Multiple focal nodular hyperplasia of the liver asso- 
ciated with congenital absence of the portal vein. Gastroenterol Clin Biol 30: 310-313, 2006 (in French, Abstract in English).

9. Takagaki K, Kodaira M, Kuriyama S, et al. Congenital absence of the portal vein complicating hepatic tumors. Intern Med 43: 194198, 2004.

10. Pichon N, Maisonnette F, Pichon-Lefievre F, Valleix D, Pillegand B. Hepatocarcinoma with congenital agenesis of the portal vein. Jpn J Clin Oncol 33: 314-316, 2003.

11. Tsuji K, Naoki K, Tachiyama Y, et al. A case of congenital absence of the portal vein. Hepatol Res 31: 43-47, 2005.

12. Grazioli L, Alberti D, Olivetti L, et al. Congenital absence of portal vein with nodular regenerative hyperplasia of the liver. Eur Radiol 10: 820-825, 2000.

13. Kanamori Y, Hashizume K, Kitano Y, Sugiyama M, Motoi T, Tange T. Congenital extrahepatic portocaval shunt (Abernethy type 2), huge liver mass, and patent ductus arteriosus-a case report of its rare clinical presentation in a young girl. J Pediatr Surg 38: E15, 2003.

14. Tanaka Y, Takayanagi M, Shiratori Y, et al. Congenital absence of portal vein with multiple hyperplastic nodular lesions in the liver. J Gastroenterol 38: 288-294, 2003.

15. Turkbey B, Karcaaltincaba M, Demir H, Akcoren Z, Yuce A, Haliloglu M. Multiple hyperplastic nodules in the liver with congenital absence of portal vein: MRI findings. Pediatr Radiol 36: 445-448, 2006.

16. Wanless IR, Mawdsley C, Adams R. On the pathogenesis of focal nodular hyperplasia of the liver. Hepatology 5: 1194-1200, 1985.

17. Kondo F. Benign nodular hepatocellular lesions caused by abnormal hepatic circulation: etiological analysis and introduction of a new concept. J Gastroenterol Hepatol 16: 1319-1328, 2001.

18. Starzl TE, Francavilla A, Halgrimson CG, et al. The origin, hormonal nature, and action of hepatotrophic substances in portal venous blood. Surg Gynecol Obstet 137: 179-199, 1973.

19. Francavilla A, Starzl TE, Porter K, et al. Screening for candidate hepatic growth factors by selective portal infusion after canine Eck's fistula. Hepatology 14: 665-670, 1991.
20. Grün R, Grün M, Rolfs A, Kaffarnik H. Hyperestrogenemia following various shunt operations: on the role of estrogens in the development of focal nodular hyperplasia of the liver. Z Gastroenterol 27: 378-382, 1989 (in German, Abstract in English).

21. Pain JA, Gimson AE, Williams R, Howard ER. Focal nodular hyperplasia of the liver: results of treatment and options in management. Gut 32: 524-527, 1991.

22. Eisenfeld AJ, Aten R, Weinberger M, Haselbacher G, Halpern K, Krakoff L. Estrogen receptor in the mammalian liver. Science 191: 862-865, 1976.

23. Rasenack U. Changes in the liver and brain after portacaval and modified portacaval end-to-side anastomosis: histology, autoradiography and clinical studies. Fortschr Med 99: 107-112, 1981 (in German, Abstract in English).

24. Takamura M, Mugishima $H$, Oowada M, Harada K, Uchida $T$. Type Ia glycogen storage disease with focal nodular hyperplasia in siblings. Acta Paediatr Jpn 37: 510-513, 1995.

25. Sakatoku H, Hirokawa Y, Inoue M, Kojima M, Yabana T, Sakurai M. Focal nodular hyperplasia in an adolescent with glycogen storage disease type I with mesocaval shunt operation in childhood: a case report and review of the literature. Acta Paediatr Jpn 38: 172$175,1996$.

26. Bjorneboe M, Andersen JR, Christensen U, Skinhoj P, Jensen OM. Does a portal-systemic shunt increase the risk of primary hepatic carcinoma in cirrhosis of the liver? Scand J Gastroenterol 20: 5964, 1985.

27. Banares R, Nunez O, Escudero M, et al. Patients with cirrhosis and bare-stent TIPS may have increased risk of hepatocellular carcinoma. Hepatology 41: 566-571, 2005.

28. Webster MW, Van Thiel DH, Bron KM, Barnes EL. Hepatic adenoma associated with portasystemic shunting in a young woman. Digestion 19: 328-334, 1979.

29. Desai S, Prabhu SR, Shrividya S. Fibrosing necrotic nodule of the liver. Indian J Gastroenterol 14: 23-24, 1995.

30. Lu SN, Lin TM, Chen CJ, et al. A case-control study of primary hepatocellular carcinoma in Taiwan. Cancer 62: 2051-2055, 1988.

(C) 2007 The Japanese Society of Internal Medicine http://www.naika.or.jp/imindex.html 